\title{
THE CHARACTERISTICS OF JUVENILE IDIOPATHIC SCOLIOSIS AND RESULTS OF ITS TREATMENT
}

\author{
VERNON T. TOLO, ROBERT GILLESPIE
}

\author{
From The Hospital for Sick Children, Toronto
}

\begin{abstract}
Fifty-nine children with juvenile idiopathic scoliosis have been reviewed. In six of eighteen who had no treatment the curvatures did not increase over eighteen months or more, while in twelve they increased at a median annual rate of six degrees. The pattern and magnitude of the curvature and the age at diagnosis could not be used accurately to predict progression. In forty-two patients progression occurred and Milwaukee brace treatment was begun, thirty being managed with periods of part-time wear. The median correction was 13 per cent. Serial measurements of the rib-vertebra angle difference (RVAD) were useful to predict a tendency to progress. Part-time wear of the brace was successful in cases where the RVAD values fell towards zero or became negative with treatment. Sixteen patients required surgical treatment; all had high positive RVAD values. However, with the plan of brace treatment outlined, part-time wear controlled many of the curvatures.
\end{abstract}

For infantile and adolescent idiopathic scoliosis the many characteristics of spinal deformity have been established so clearly that a typical case of either condition can be easily portrayed. Between these two well-defined groups lies that of juvenile idiopathic scoliosis, which includes children who are four to ten years of age at the time of diagnosis and about whom less is known. Whether enough distinctions make this a separate group is not clear. Indeed, in her review of idiopathic scoliosis, Wynne-Davies (1968) deleted the juvenile group altogether, divided these patients between the infantile and adolescent groups, and used the age of eight to define "early-onset" and "late-onset" groups. The Scoliosis Research Society, however, recognises the juvenile category as a specific age group.

Less common than the scoliosis of adolescence, juvenile idiopathic scoliosis presents special problems in management. If bracing is commenced it must usually be maintained for several years, and if operation is performed in early childhood, the outcome of a short trunk is often disappointing. With little information reported on this group, our purpose in this review was to attempt to establish its characteristics, to identify any feature useful in determining the prognosis, and to assess the efficacy of our regime of brace treatment.

\section{METHODS}

The fifty-nine patients included in the study were all treated either at the Hospital for Sick Children in Toronto in the Orthopedic or Brace Clinic, or in the private practice of the senior author (R.G.). All children with idiopathic scoliosis diagnosed between the ages of four and ten were reviewed. The curve patterns were classified according to the level of the apical vertebra: high thoracic, above T5; thoracic, between T6 and T10; thoracolumbar, T11 or T12; and lumbar, L2 or L3. If two structural curves were present the double curve was called asymmetrical if one was larger than the other, and symmetrical if they were about equal. All radiographs were taken with the patient standing without a brace,

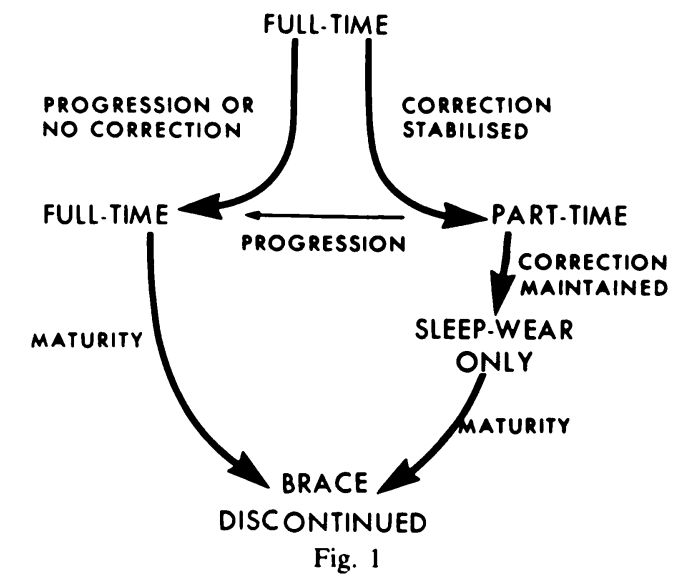

Showing the present plan of treatment by a Milwaukee brace for juvenile idiopathic scoliosis.

and curves were measured by the Cobb method. Rotation was determined by the method of Nash and Moe (1969). The rib-vertebra angle difference (RVAD) was measured as described by Mehta (1972); in a curve with an odd number of vertebrae, the middle vertebra was used for this measurement; otherwise the lower of the two middle vertebrae was chosen. 
For the follow-up study each patient was placed in one of three groups: no treatment, treatment with a Milwaukee brace, or operative treatment. Some were included in more than one group because their treatment was changed according to the behaviour of the curve.

In Group I serial radiographs were obtained at intervals of four to twelve months but no treatment was given. For Group II the plan of brace treatment is illustrated in Figure 1. Full-time wear, with two hours free daily, was commenced initially and usually continued for at least a year. The exercises described by Blount and Moe (1973) were an essential part of the treatment, and general physical activity was encouraged. Radiographs were taken at intervals of four months, when the exercise performance was reviewed by a physiotherapist. Part-time wear, the brace being discarded for four hours or more daily, was begun once the curve had apparently stabilised. Provided no progression resulted, the period of wear was decreased by twoto four-hour increments until the brace was worn only during the hours of sleep. The use of a brace was continued until growth was complete.

\section{RESULTS}

Of the fifty-nine patients, forty-eight were girls and eleven boys, a ratio of 4.4 to 1 . In the children aged four to six, the ratio was almost equal, while there were no boys at all among those aged seven to eight. In the children of nine and ten the ratio was 8.7 to 1 .

At the time of diagnosis a single thoracic curve (27) was most common, followed by double curves (19), lumbar (7), thoracolumbar (4), and triple curve patterns (2) (Fig. 2). Of the twenty-seven single thoracic curves,

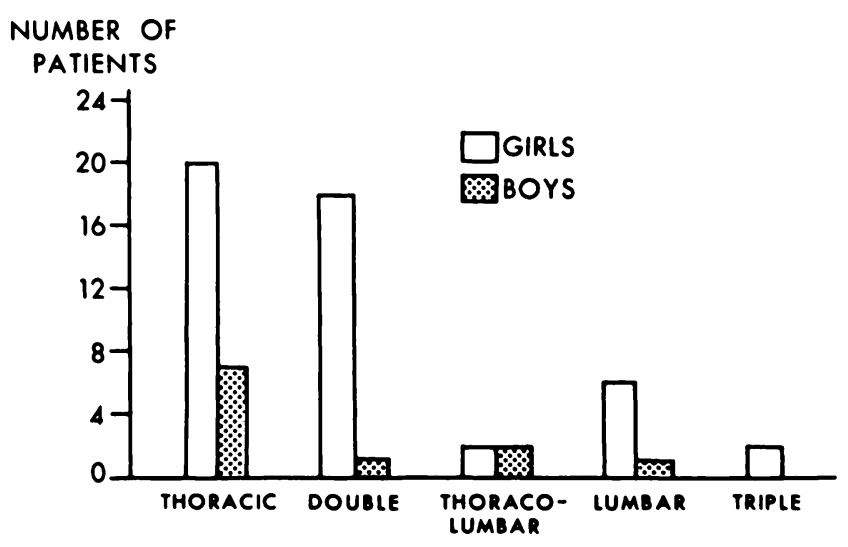

Fig. 2

Showing the distribution of curve patterns in girls and boys at the time of diagnosis.

three were to the left. Most of the double curves were right thoracic, left lumbar. All the lumbar curves were to the left, and all the thoracolumbar curves were to the right. Of the boys, all but one had a single curve, whereas the pattern in girls was double almost as often as single. Before the age of eight the double curves were often asymmetrical with the thoracic curve larger than the lumbar, while in the older children symmetry was more common. Single lumbar curves were not seen before the age of nine (Fig. 3).

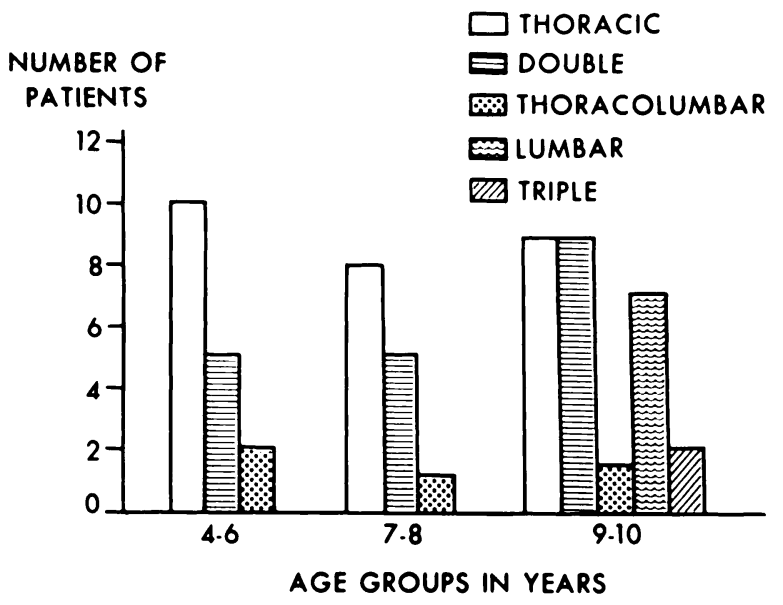

Fig. 3

Showing the distribution of curve patterns by age.

Group I. Serial radiographs were available on eighteen patients who received no treatment over periods ranging from sixteen to sixty-four months. Of these, six showed no appreciable increase, namely, less than three degrees, whereas twelve progressed. Two of the twelve had had periods of no progression of fifteen and twenty-four months respectively. Of the curves that increased, the median annual rate of progression was 6.2 degrees overall (Fig. 4), the most being in single thoracic curves

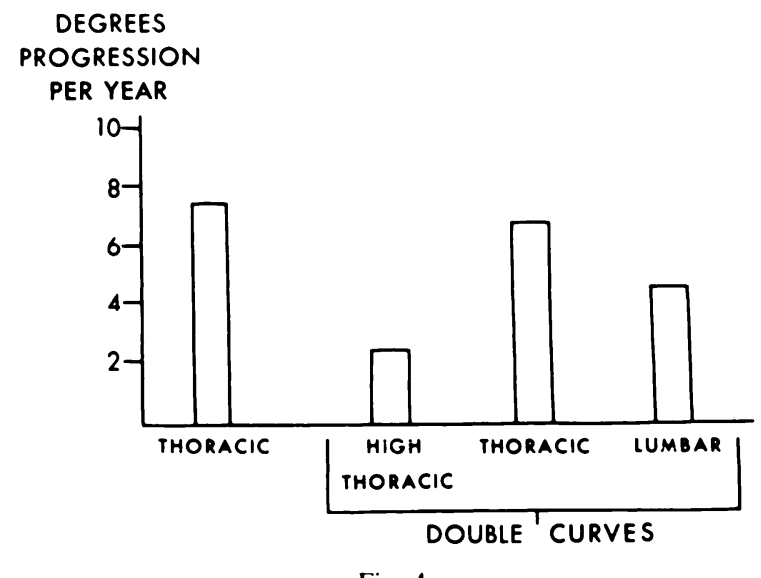

Fig. 4

Group I. Showing the median annual rates of progression of untreated curves observed in twelve of the eighteen patients.

(7.3 degrees). Double and triple pattern curves progressed at slower rates, with a right thoracic curve tending to progress more rapidly than the others (Fig. 5). Curves in children with siblings also showing scoliosis were among 

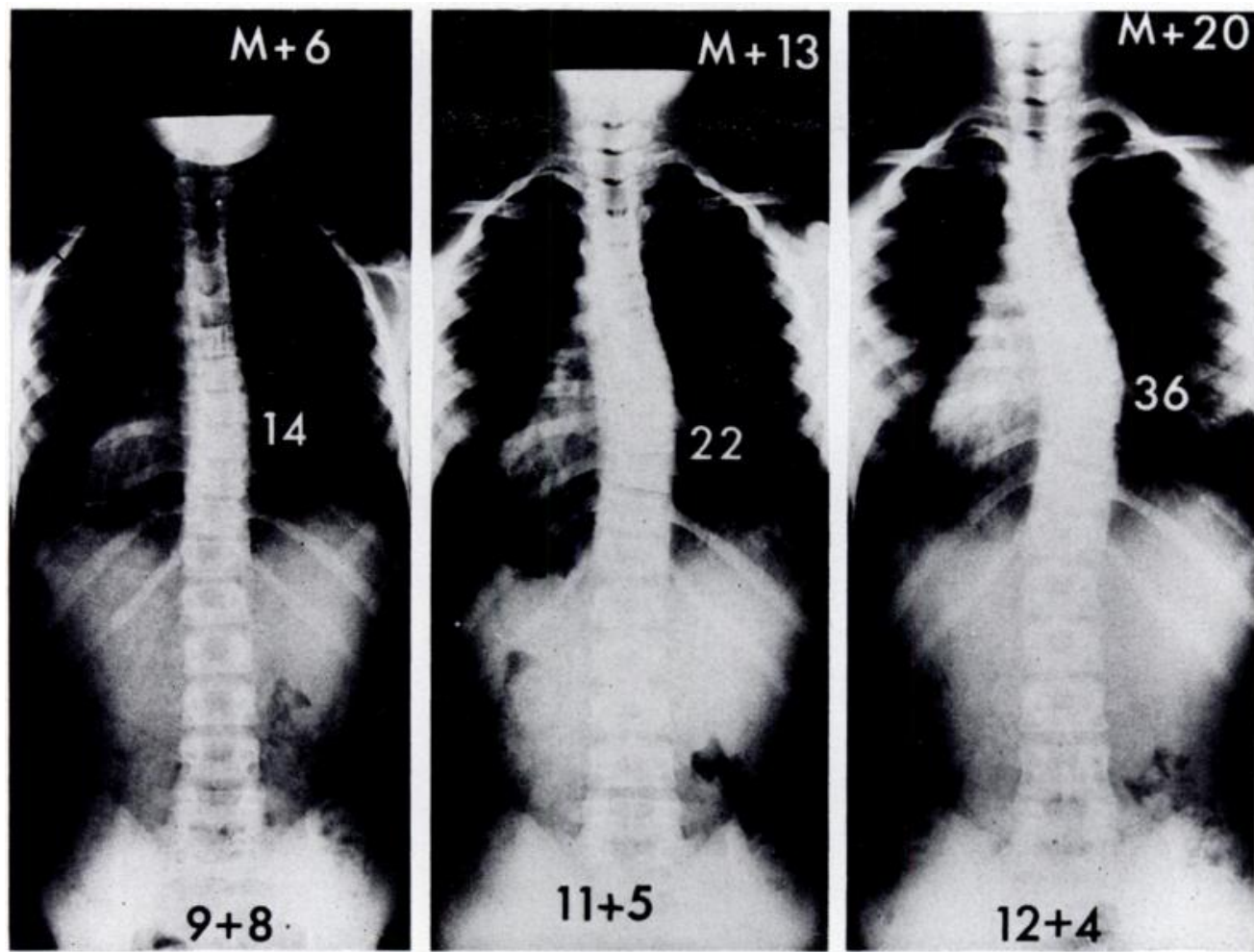

Fig. 5

Serial radiographs of a girl under no treatment, showing progression of a thoracic curve.*
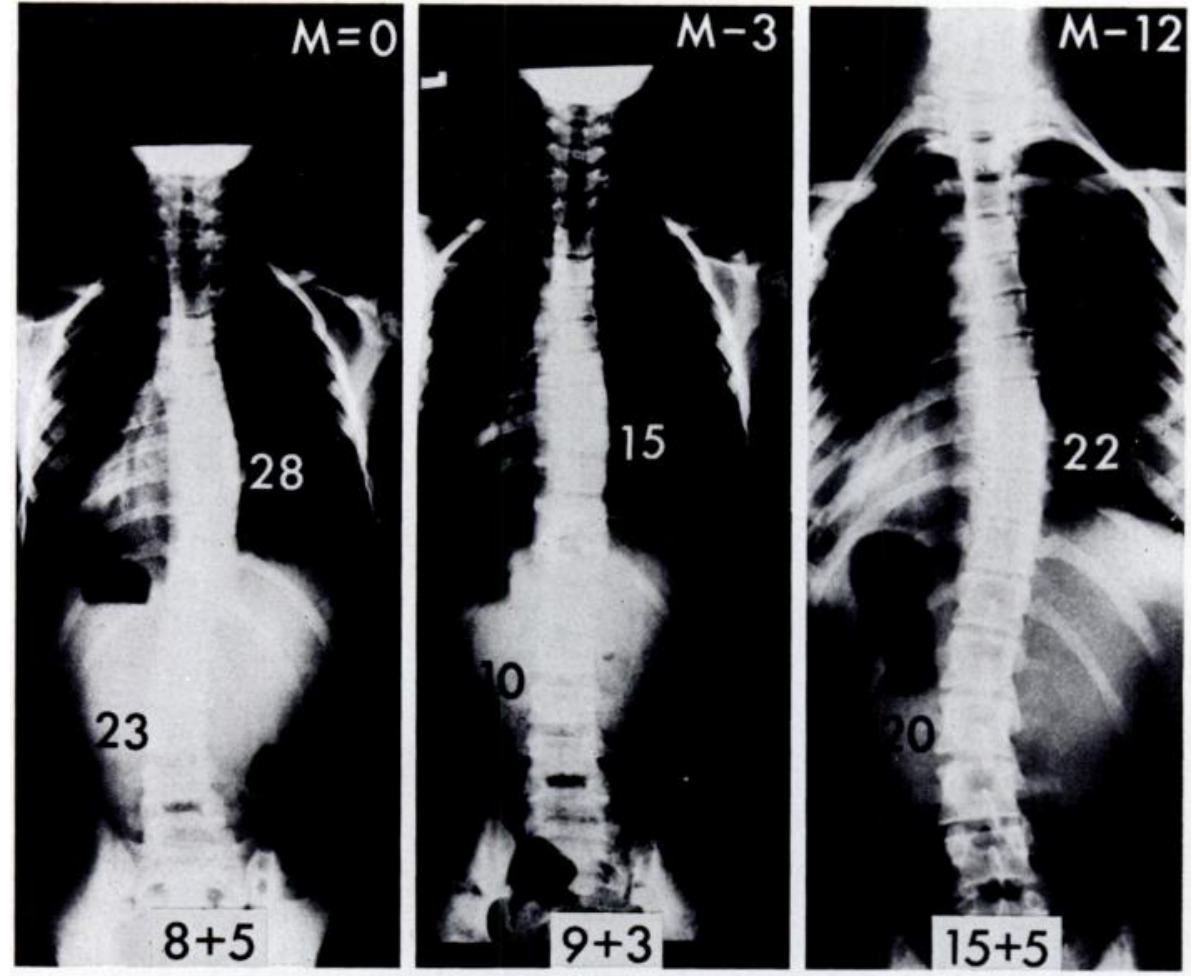

Fig. 6

Radiographs of a girl effectively treated largely by part-time brace wear. She wore the brace full-time for nine months, then part-time for almost six years, including night wear only after the age of ten.

"Note that in all the present radiographs, the age is noted below in years + months, then the magnitude of the curve in degrees, and at the top the rib-vertebra angle difference of Mehta (M). Note also that all the radiographs are shown as postero-anterior views. 
the most progressive. The number of vertebrae in the curve could not be used to predict progression except in some curves with nine or ten vertebrae, which did not increase during the period of review. Measurements of rotation were of little help with prognosis, as they followed or coincided with the magnitude of the curves.

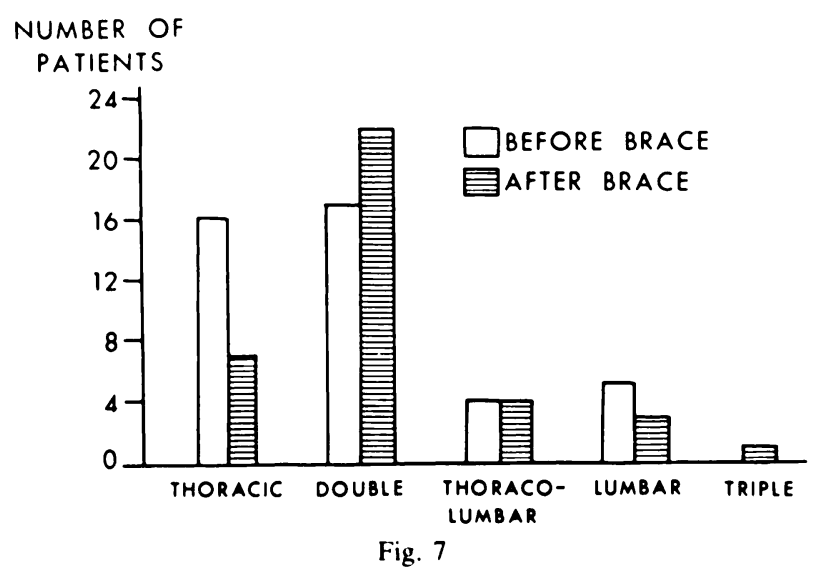

Group II. The distribution of curve patterns before and after brace treatment. showing an increase in double curves.

The median changes in RVAD were not markedly different for cases that did not progress ( -3 degrees) from those that did ( +4 degrees). Five out of six patients with an initial RVAD of more than +5 degrees progressed, as did both of those with a value over +10 degrees. Nine of the cases of progression had RVAD values that became increasingly positive with time. Three-quarters of the patients without progression had a steady reduction in RVAD values.
Group II. Milwaukee brace treatment was used in forty-two patients, on average for three years. Thirtyfive of these patients wore a brace for more than one year but only six full-time; the remainder were allowed at least some part-time wear during the juvenile age period. The seven patients reviewed who wore a brace

Table I. The marked variation of amount of correction by bracing in different patterns of curvature

\begin{tabular}{|c|c|c|c|}
\hline $\begin{array}{c}\text { Curve } \\
\text { pattern }\end{array}$ & $\begin{array}{c}\text { Number of } \\
\text { curves }\end{array}$ & $\begin{array}{c}\text { Median } \\
\text { best } \\
\text { (per cent) }\end{array}$ & $\begin{array}{c}\text { Correction at } \\
\text { final review } \\
\text { (per cent) }\end{array}$ \\
\hline $\begin{array}{c}\text { Single thoracic } \\
\text { Single lumbar }\end{array}$ & 16 & 37 & 6.5 \\
Thoracolumbar & 4 & 23 & 13 \\
Double curves \\
Thoracic
\end{tabular}

for less than a year (five to twelve months) were all on a full-time schedule. That part-time brace wear can be used successfully for prolonged periods is illustrated by the radiographs in Figure 6 . Brace treatment is continuing on all of these patients except four who have reached maturity and eight who have had operations.

Single curves were more common before brace treatment, but there was a majority of double curves after treatment (Fig. 7). Curves initially single thoracic
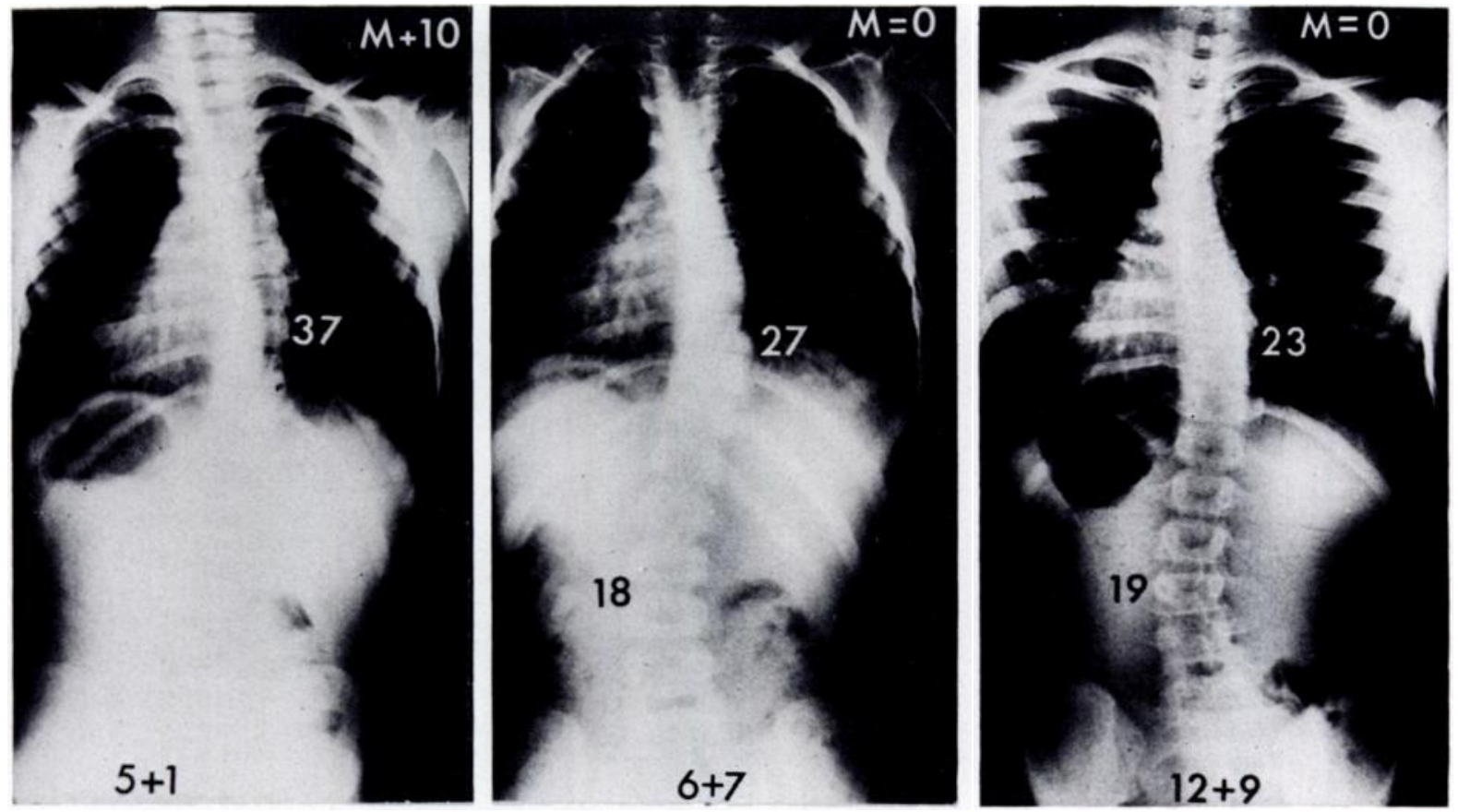

Fig. 8

Radiographs of a boy illustrating the tendency of a single curve to become double with bracing. 
or lumbar tended to become double patterns with the brace (Fig. 8). The range of percentage loss or correction with the brace is shown in Figure 9; overall, the median was 13 per cent correction at final review (Table I). Of those patients treated for more than a year, one half had their best correction by eight months, while a quarter did not attain this for two years or more. Single thoracic

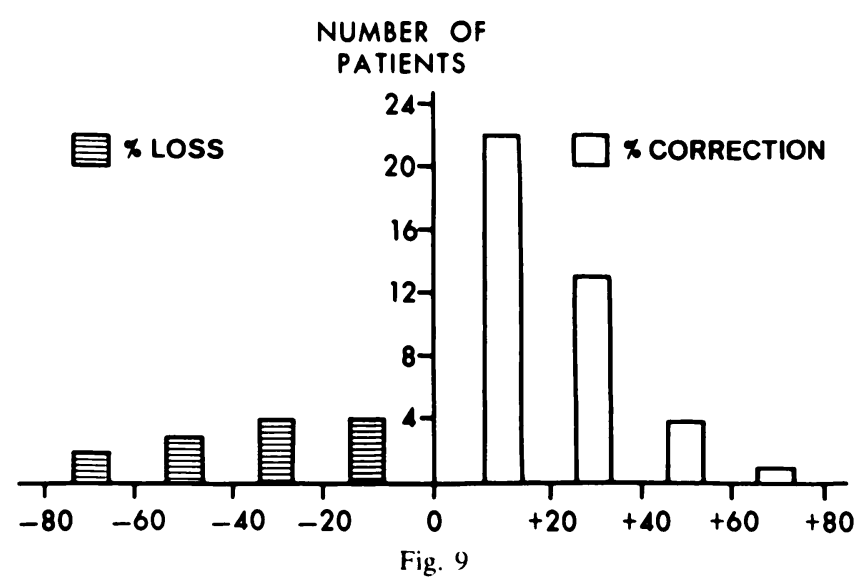

Group II. Showing the distribution of percentage loss or correction by bracing.

curves often had the best initial correction but gradually regressed (Table $\mathrm{I}$ ). The thoracolumbar group, though small, had the best response of all curve patterns, as illustrated by the patient in Figure 10. Patients with eight to ten vertebrae in the curve had a median correction of 24 per cent, while shorter curves responded less well.
Curves of four to five vertebrae had 12 per cent correction and those of six to seven had 8 per cent.

Group III. Surgical treatment was used in sixteen patients, fourteen having Harrington rod instrumentation and spinal fusion and two electrospinal instrumentation, an approach developed by $\mathrm{Dr}$ W. Bobechko in Toronto. Half of the sixteen had brace treatment initially, while the other half underwent an operation as primary treatment. Of the seven with primary Harrington rod instrumentation and fusion, thoracic curves measured 36 to 65 degrees with a median of 50 degrees, and rotation was +2 or +3 in all of them. The median age at operation was 9.7 years. The RVAD values were all positive and high, with a range of +12 to +30 degrees and a median of +27 degrees.

All seven of those patients treated with a brace before Harrington instrumentation were girls. Four had single thoracic curves and three had double curves. An average of four years passed between diagnosis and operation, when the median age was 12.5 years. Before brace treatment all RVAD values were positive, with a median of +16 . Part-time wear was used in five out of seven of these patients; in some this was allowed because an operation was predicted to be needed at a relatively early age but delayed to allow more growth of the trunk. Lack of success of brace treatment. This occurred for several reasons. Four girls would not accept a brace. One girl was prematurely removed from brace wear and fusion was performed at the age of eleven when her

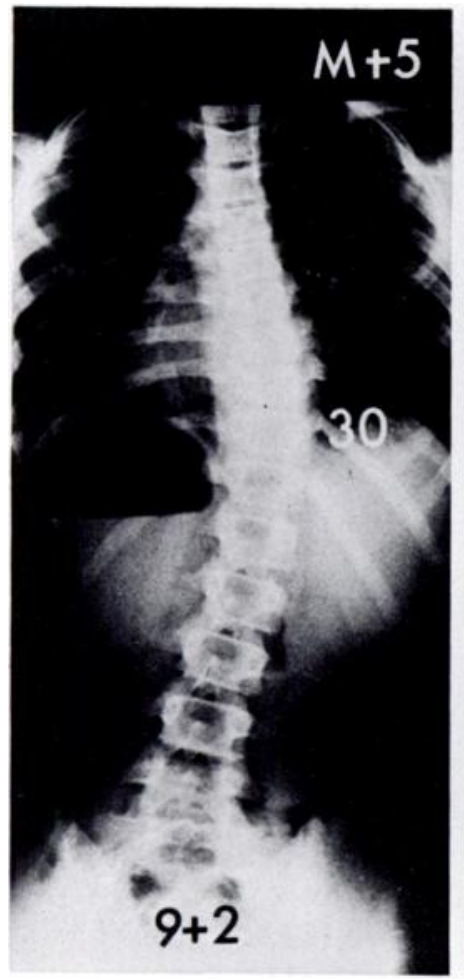

Radiographs of a girl showing excellent control of a long thoracc

Fig. 10
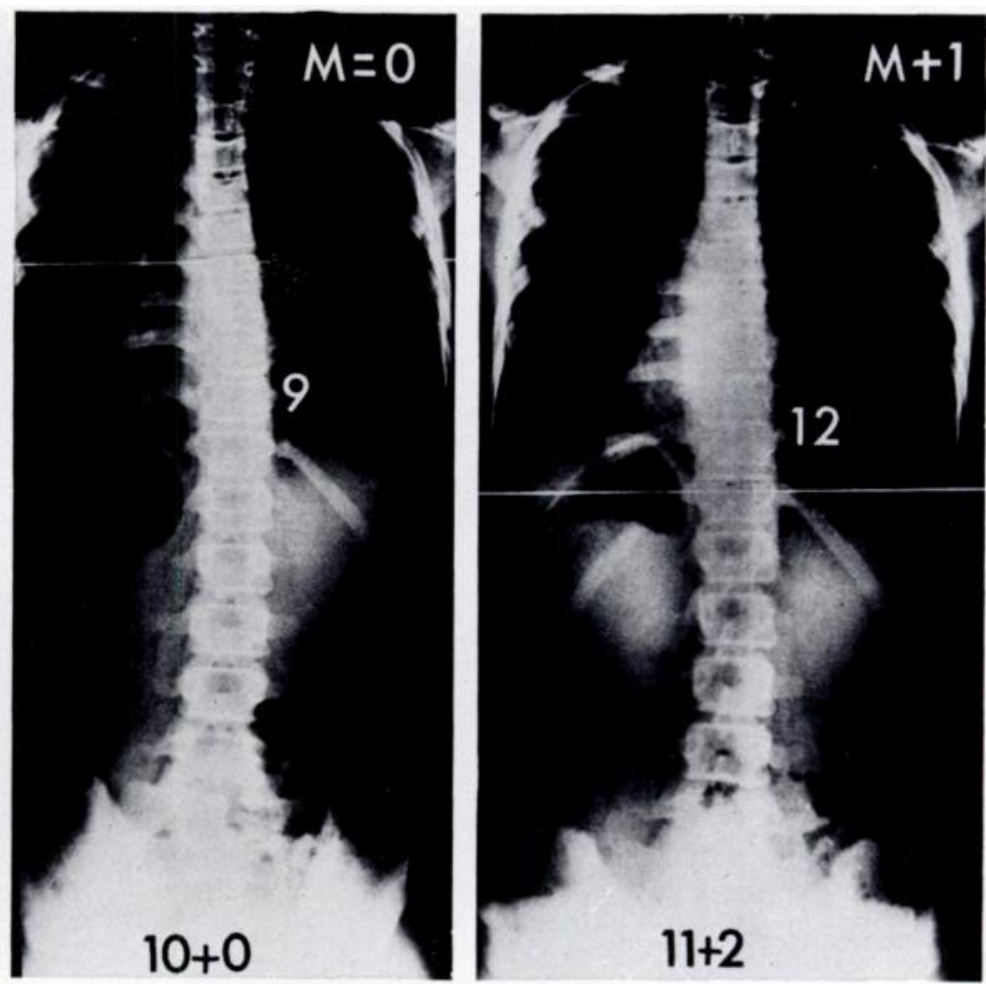
months of full-time wear. 

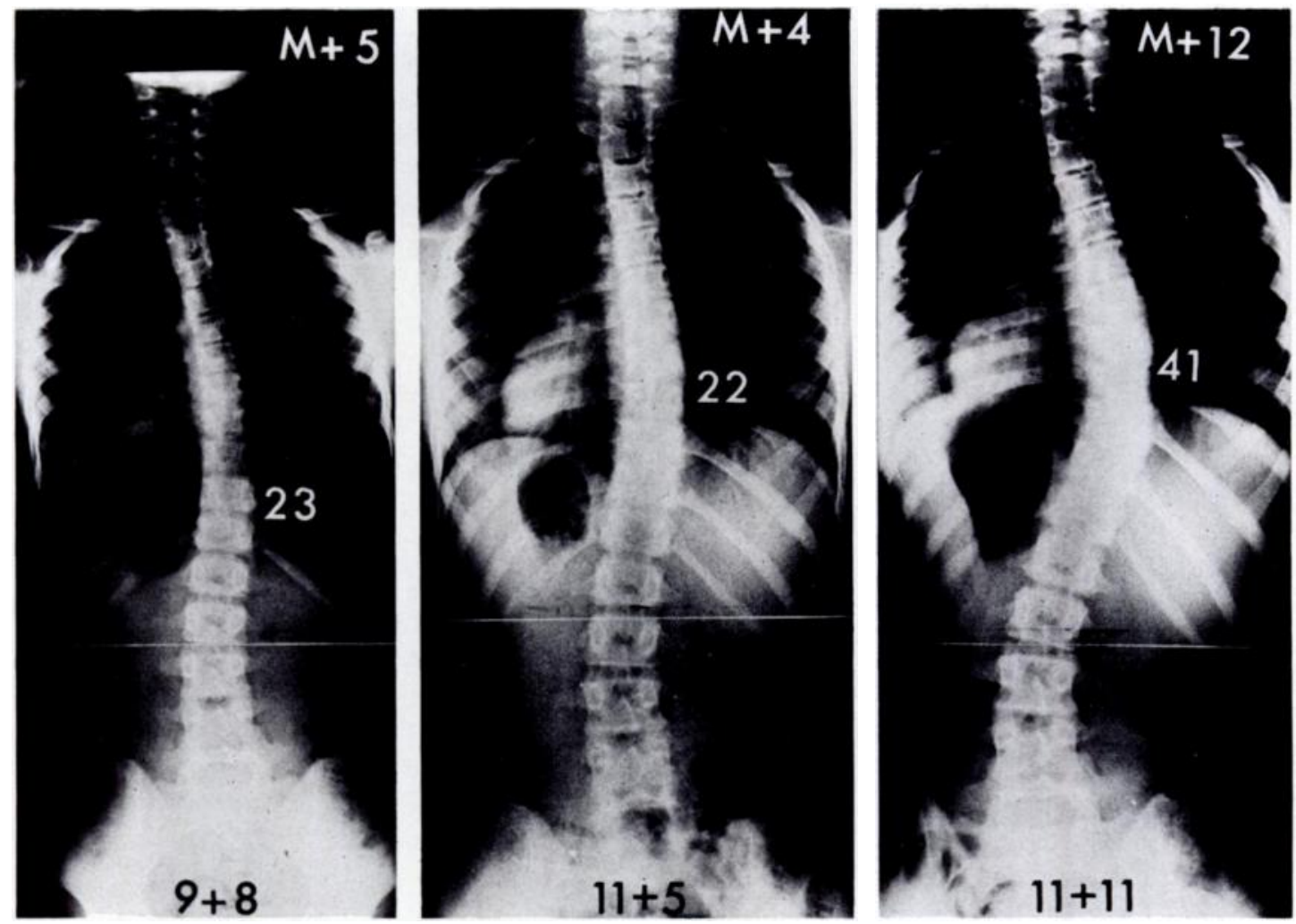

Fig. 11

Radiographs of a girl with a curve, initially non-progressive, that progressed quickly when brace wear was temporarily discontinued during the period of adolescent growth.
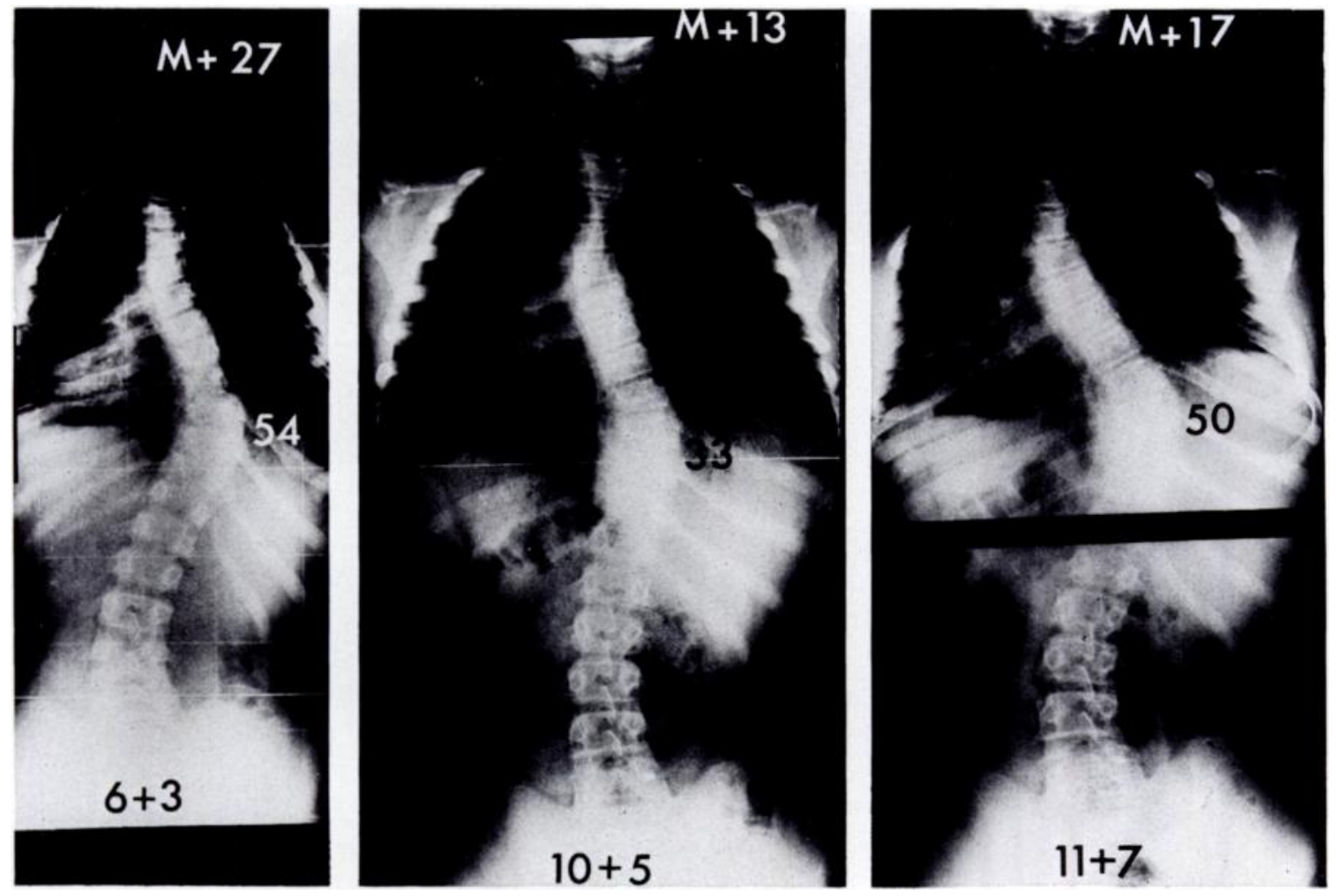

Fig. 12

Radiographs showing persistently high RVAD values and progression of the curve despite temporary improvement. 


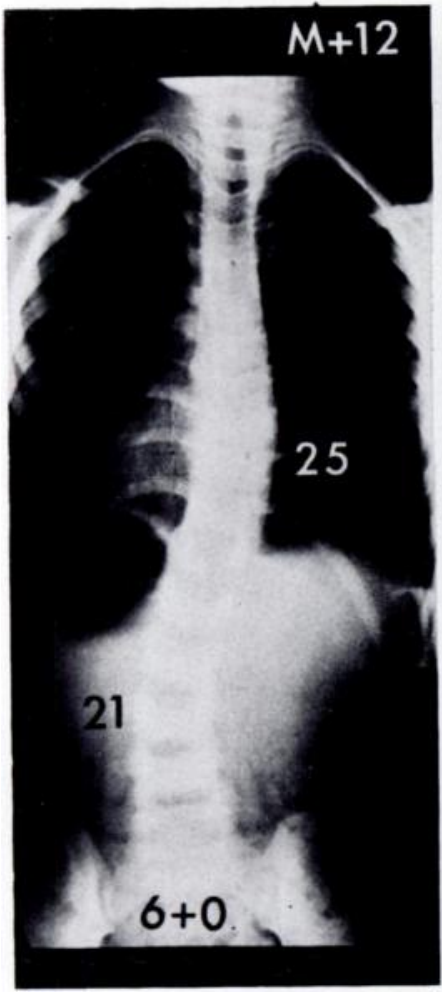

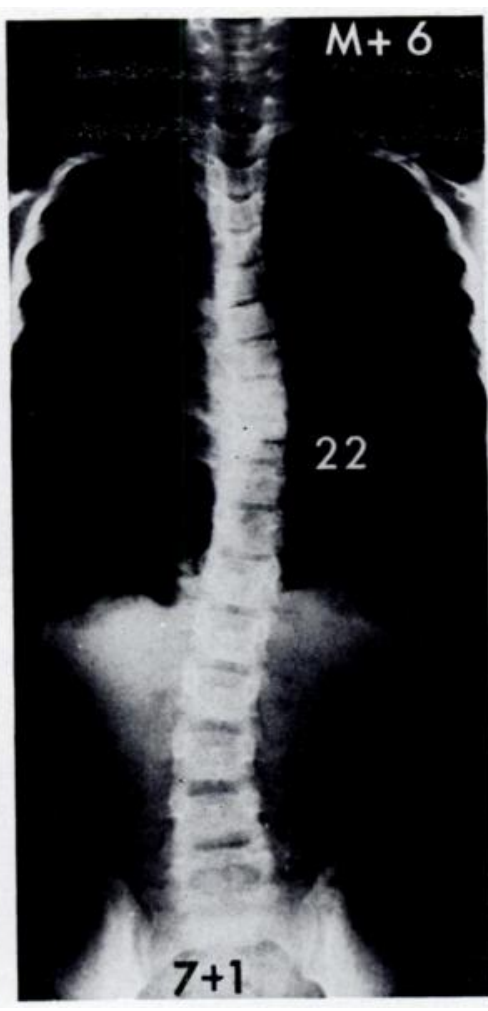

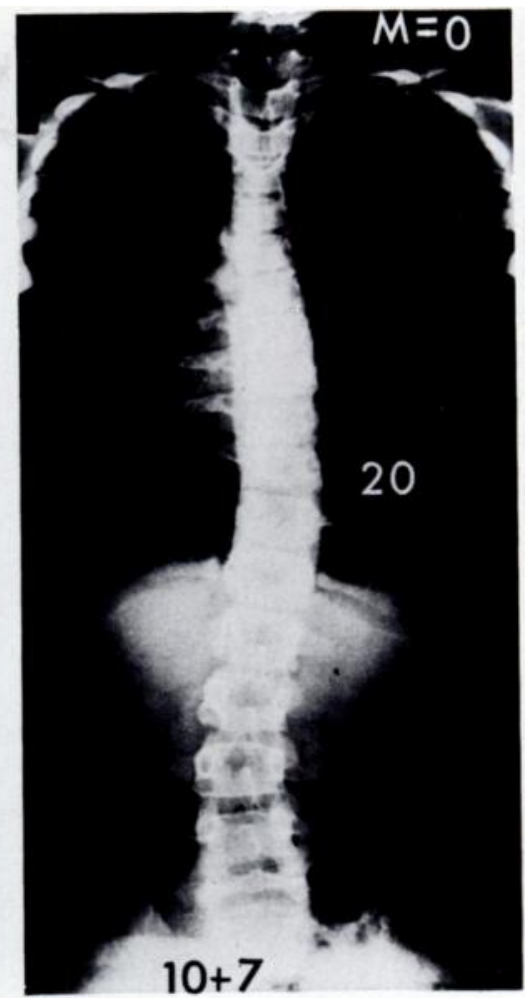
the brace.

height was already 70 inches (178 centimetres), while another had a double curve of almost 50 degrees when brace treatment was started. The last girl, after a period of part-time wear had led to no progression, was left out of her brace for six months at the age of eleven, but over the next six months the curvature increased from 22 degrees to 41 degrees (Fig. 11), and renewed bracing failed to stop progression.

All of these patients with a poor response to bracing had persistent RVAD values of +10 degrees or more, despite transient improvement in the curves (Fig. 12). Similar curve patterns in those successfully treated with a Milwaukee brace showed gradually decreasing RVAD readings towards zero and even negative values (Fig. 13).

\section{DISCUSSION}

With modern techniques the management of adolescent scoliosis has become straightforward, and in our view the problems now lie principally with the infantile and juvenile groups. The significance of small curves is difficult to determine and thus the rigidity with which a brace-wearing schedule should be imposed on a young child is uncertain. Effective screening should change the pattern of referral towards a time when orthopaedic surgeons see a larger number of younger children with smaller curves and fewer adolescents or adults requiring primary operation. Furthermore, the poor results oblained in those few children with relentlessly progressive curves who come to operation before the age of ten is frustrating in that all too often the end result is satisfactory only in terms of control of the lateral curvature as seen on the radiograph, the patient being left with a short trunk, a marked thoracic deformity and a fused chest. By learning more of the characteristics and behaviour of scoliosis in these young children we should be able to treat them more effectively without subjection to unnecessarily rigid wearing of a brace.

In reviews of idiopathic scoliosis the proportion of younger children has varied. Of the patients reviewed by Ponseti and Friedman (1950) 13 per cent were under ten years of age, while James (1954) found 15 per cent in the range of five to eight years. In their review of brace-wearing patients Moe and Kettleson (1970) had 15 per cent between three and ten years of age. From the data of Wynne-Davies (1968), the proportion of children aged four to ten years was 21 per cent. It would therefore seem that this group is sufficiently large to warrant separate consideration.

Opinions on which curve pattern predominates in this juvenile group have not been unanimous, perhaps partly due to geographical differences. Thus, in the United States Ponseti and Friedman found single curves most common under the age of ten. They also noted that in the double curves the thoracic component was usually larger than the lumbar, and that lumbar curves stabilised 
at an earlier age than did the other patterns. In Great Britain, on the other hand, James found that double curve patterns predominated and concluded that this was the characteristic pattern of the juvenile group. Noting their often asymmetrical nature, he found that the curves were usually left thoracic, right lumbar in the younger patients and more commonly right thoracic, left lumbar as the age of onset increased. In our group, single right thoracic curves were most common at an early age, when the sex ratio was equal, and at a later age, when girls predominated, the number of double curves equalled the number of single thoracic curves. In the younger patients double curves were usually asymmetrical.

From the curve pattern, the degree of curvature, or the age at the time of diagnosis, it was not possible to predict which curves would increase and which would not. In this respect the initial RVAD measurement was of only limited value compared with serial readings. We believe that unless the curve is quite advanced when the juvenile patient is first seen, evidence of progression should be obtained before a Milwaukee brace is applied, because in some cases, even in the range of 20 to 30 degrees, no progression occurred over a period of several months. Once progression is proven, the Milwaukee brace is a remarkably effective device.

The RVAD does appear useful when measured serially. In the group treated with the Milwaukee brace, all single curves that progressed had values that were increasingly positive as time went on. In some cases this increase preceded an increase in magnitude of the curve by a few months. As for the more benign curves-single and double curve patterns that did not progress, as well as single curves that became double patterns-all the changes in RVAD were towards zero or even negative values. With brace treatment, if the RVAD did not go below +10 degrees progression could be expected.

If the RVAD values decline as treatment continues, part-time wear of a brace should be adequate. Those curves with RVAD values near or below zero at the time of diagnosis will generally be able to move to part-time wear after a relatively short period of full-time wear. Even those with significant times free of progression, however, must be carefully observed to maturity in order to detect any relapse when the rate of growth increases around puberty.

Rapport with the child and his parents, careful periodic examination, good orthotic craftsmanship, and the performance of regular exercises in and out of the brace-all these are necessary components of Milwaukee brace treatment, whether part-time or full-time. Attention to these details, and to the guidelines proposed above to differentiate progressive from more stable curves, can often allow these juvenile curves to be treated successfully with a schedule of bracing that includes periods of part-time wear.

\section{REFERENCES}

Blount, W. P., and Moe, J. H. (1973) The Milwaukee Brace. Baltimore: The Williams and Wilkins Company.

James, J. I. P. (1954) Idiopathic scoliosis. Journal of Bone and Joint Surgery, 36-B, 36-49.

Mehta, M. H. (1972) The rib-vertebra angle in the early diagnosis between resolving and progressive infantile scoliosis. Journal of Bone and Joint Surgery: 54-B, 230-243.

Moe, J. H., and Kettleson, D. N. (1970) Idiopathic scoliosis. Journal of Bone and Joint Surgery: 52-A, 1509-1533.

Nash, C. L., Jun., and Moe, J. H. (1969) A study of vertebral rotation. Journal of Bone and Joint Surgery, 51-A, 223-229.

Ponseti, I. V., and Friedman, B. (1950) Prognosis in idiopathic scoliosis. Journal of Bone and Joint Surgeri: 32-A, 381-395.

Wynne-Davies, R. (1968) Familial (idiopathic) scoliosis. Journal of Bone and Joint Surgery, 50-B, 24-30. 